\title{
Mito-nuclear selection induces a trade-off between species ecological dominance and evolutionary lifespan
}

\section{Debora Princepe ( $\sim$ princepe@ifi.unicamp.br)}

Universidade Estadual de Campinas

\section{Marcus Aguiar}

Universidade Estadual de Campinas

Joshua Plotkin

University of Pennsylvania https://orcid.org/0000-0003-2349-6304

\section{Article}

Keywords: Micro-evolutionary processes, spatially extended populations, mito-nuclear compatibility

Posted Date: November 29th, 2021

DOI: https://doi.org/10.21203/rs.3.rs-1061315/v1

License: (1) This work is licensed under a Creative Commons Attribution 4.0 International License.

Read Full License

Version of Record: A version of this preprint was published at Nature Ecology \&amp; Evolution on October 10th, 2022. See the published version at https://doi.org/10.1038/s41559-022-01901-0. 


\title{
Mito-nuclear selection induces a trade-off between species ecological dominance and evolutionary lifespan
}

\author{
Débora Princepe, Marcus A. M. de Aguiar and Joshua B. Plotkin
}

Micro-evolutionary processes acting in populations and communities ultimately produce macro-evolutionary patterns. However, current models of species life histories - including processes of speciation, persistence, hybridization, and eventual extinction - rarely connect these two time scales. This leaves us with a limited theoretical understanding of the subtleties of diversification, such as the relationship between species abundance in an ecological community and species longevity over evolutionary time, or the impact of selection on patterns of speciation and extinction when structuring an ecological community. Here we present a model for evolution in spatially extended populations with a focus on selection for mito-nuclear compatibility. We find that mito-nuclear selection acting at the individual level decreases genetic variability among species in a radiation, reducing the total number of species and skewing species abundances distributions towards mono-dominance. Also, intraspecific diversity increases as species become more abundant, leading to frequent evolutionary branching that reduces species lifetimes. The equilibrium of such communities is characterized by high rates of speciation, extinction, and hybridization, i.e., high turnover rate. These theoretical results are in concordance with empirical patterns of diversity across latitudinal gradients. Model predictions in the absence of mito-nuclear selection resemble the tropics, with high biodiversity, old species, and low speciation and extinction rates. Whereas model predictions under strong selection, which we expect in the harsh environments of temperate zones, produce fewer species and elevated recent speciation rates. 


\section{Introduction}

The origin, maintenance, and extinction of species are subjects typically studied separately, as they occur on different time scales. Mathematical models have been helpful to explain fundamental patterns of biodiversity, such as species abundance distributions [1-4] and the species-area curve $[5,6]$, supported by both the neutral theory and by the niche theory of community assembly $[7,8]$. However, models that explain such ecological features often fail to predict speciation rates [9] and species lifetimes [10-12], and they do not provide a mechanistic description of the formation of species $[13,14]$. In this context, understanding the connection between ecological and evolutionary aspects of how biodiversity is assembled can help to explain phenomena that depend on the interaction between them, for example, how species life histories are associated with their commonness or rarity $[15,16]$.

An important open problem that requires such integrative perspective is to understand the latitudinal gradient of species, that is, the increase of species richness towards the equator $[17,18]$. There is no consensus about the causes of the richness gradient [19], and, moreover, there is puzzling empirical pattern than the tropics show reduced recent speciation rates despite having a greater number of species [20-25]. Insight into this surprising observation can be gleaned from models of protracted speciation, which describe a population splitting and the sequential evolution of reproductive barriers, which may differ along the gradient and cause a higher speciation rate in temperate zones. Environmental harshness may also play a role in the ephemeral persistence of species is such an ecological speciation process [26]. This perspective suggests the need to integrate population dynamics and macroevolutionary theory.

More generally, biotic and abiotic factors affect the diversification process, which reflects a balance between speciation and extinction events [27]. Studying the impact of these factors on the emergence and disappearance of species is, therefore, essential to identify signatures that these processes could imprint at the evolutionary scale $[28,29]$. Furthermore, population genetics can help elucidate how these processes connect across different scales, such as how selection at the individual level can lead to genetic diversity and evolution of reproductive barriers $[26,30-32]$. In this context, considering micro-evolutionary processes when analyzing macro-evolutionary patterns is necessary to fully understand the underlying mechanisms driving diversification. This is especially true in light of evidence that similar evolutionary signatures can result from different processes in populations $[33,34]$, and that generalized birth-death models can produce 
extant trees that correspond to countless different diversification processes [35]. Models that explicitly include a micro-macro link provide explanations for ecological patterns [36] while also elucidating signatures in phylogenetic trees [37].

Here we present an investigation of population dynamics that connect with micro-ecological patterns and ultimately leave macro-evolutionary signatures. We study spatially distributed populations that evolve under selection for mito-nuclear compatibility [38]. The genetic interaction between the mitochondrial and nuclear genomes is necessary for respiration, and is known to induce molecular co-evolution $[39,40]$. Mito-nuclear interactions have been implicated as a driver of speciation, where hybrid infertility results from incompatible mito-nuclear combinations [41,42]. The metabolic role of mitochondria also suggests that selection may be driven by the environment, with effects of the mito-nuclear interaction leaving signatures across biological scales - within individuals, intraspecies and interspecies [43-46]. Moreover, the mitochondrial genetic material is commonly used as an evolutionary marker, given its properties of haploidy, uniparental inheritance, and rapid evolution $[47,48]$. Therefore, the mito-nuclear interaction provides a natural connection between population genetics, ecology, and evolutionary processes. We use an individual-based model, simulating selection on mito-nuclear compatibility and following the process of radiation and eventual equilibrium towards a stationary diversity of species. These simulations record all forms of diversification events - speciation, extinction, and hybridization - and evaluate the impact of selection on the emergence and persistence of species. In addition, we assess the history of an extant community, analyzing the dominance of species throughout their evolutionary lifetime. We use this model to reveal how mito-nuclear selection can jointly influence species abundances and lifetimes, unveiling how abundance distributions connect with species history, lifespans, and the processes guiding diversification.

\section{Results}

\subsection{Model for mito-nuclear interaction}

We employed an agent-based model (Fig. 1, Methods) where each individual is described by a haploid, bi-allelic sequence of $B$ sites that represent its nuclear genome (nDNA), along with a shorter string of $B_{M}$ sites representing a copy of its mitochondrial genome (mtDNA). Sexes are separated, and sexual reproduction with non-overlapping generations occurs as follows: 


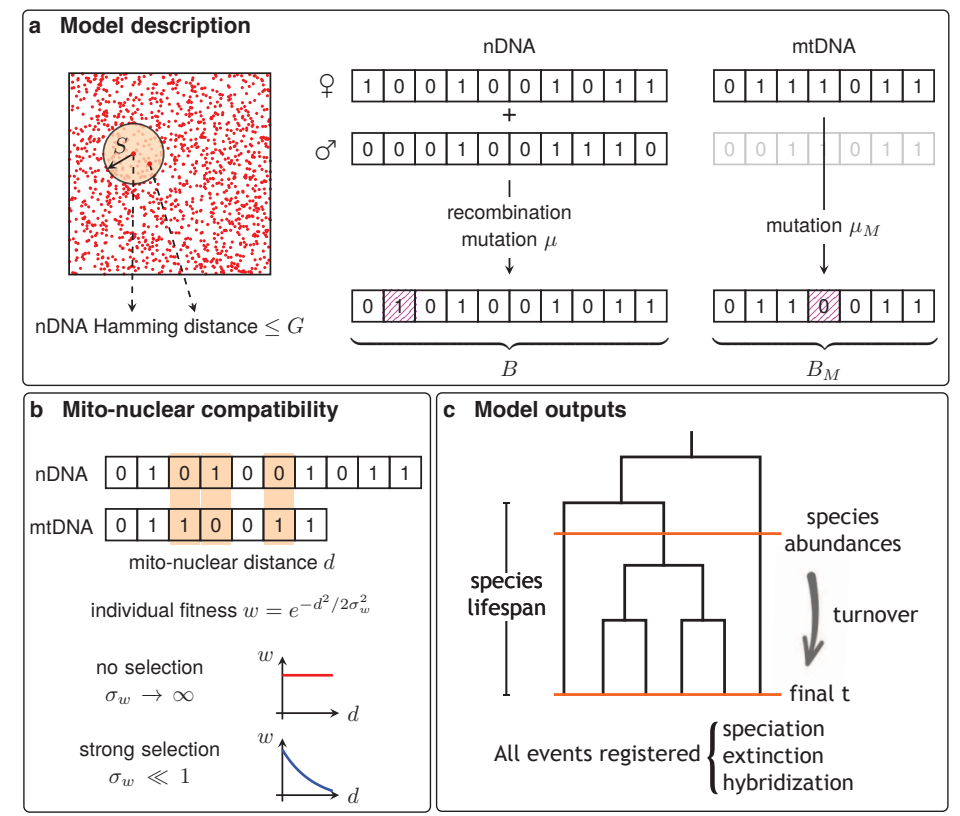

Figure 1: Model of selection on mito-nuclear compatibility. a, Individuals are randomly distributed in a square domain and carry nuclear (nDNA) and mitochondrial (mtDNA) genomes described by binary sequences of $B$ and $B_{M}$ sites, respectively. Sexual reproduction occurs with non-overlapping generations. Individuals look for a mating partner within a range of radius $S$, and they must be genetically compatible (within nuclear distance $G$ ) to mate. Offspring are generated with recombination of the nDNA and mutation probability $\mu$ per locus, with direct inheritance of maternal mtDNA with mutation $\mu_{M}$. b. Mito-nuclear compatibility of an individual is measured by the distance between the mtDNA and corresponding sites of the nDNA. In the absence of selection, all individuals have equal fitness. Under selection for mito-nuclear compatibility fitness is assigned to individuals according to their mito-nuclear distance, with strength of selection $\sigma_{w}$, with higher fitness implying higher probability to reproduce. c, Species are classified as groups of individuals with ongoing gene flow, and their abundances are recorded at each generation. We register all speciation, hybridization, and extinctions events, and record the lifespans of all species.

during the reproduction phase, all individuals have a probability to randomly choose a mating pair within a spatial range of radius $S$. Genetic compatibility between individuals is determined by the Hamming distance between their nDNA's, i.e., the number of loci bearing different alleles, and must be below the threshold of $G$ sites for successful reproduction. The nuclear genome of the offspring is built from recombination of the nuclear material of the parents, with a probability of mutation $\mu$ per site. The mtDNA is directly inherited from the mother, with mutation probability per site $\mu_{M}$. The population size is kept constant. Starting from identical individuals, mutations and genetic drift combined with spatial and genetic restrictions on mating, species emerge as clusters of genetically compatible individuals, i.e., as groups with ongoing gene flow among individuals, determined by the genetic mating restriction $G$, but no gene flow between different groups (see Methods Section 4.1).

We model mito-nuclear compatibility in an individual as a locus-by-locus interaction between the mtDNA and the first $B_{M}$ sites of the nuclear genome; the interacting pair of loci 
is compatible if they have the same allele value. The mito-nuclear distance $d$ is then the total number of incompatible sites. Such a scheme is a phenomenological description of the biochemical and structural compatibility between proteins coded by both genomes necessary for the respiration process $[43,44]$. In the absence of selection, all individuals have an equal probability of reproduction, and nDNA and mtDNA evolve independently. When mito-nuclear compatibility is under selection, fitness $w$ is assigned to individuals accordingly to their mito-nuclear distance, with strength expressed by a parameter $\sigma_{w}$, such that $w=e^{-d^{2} / 2 \sigma_{w}^{2}}$, i.e., small $\sigma_{w}$ implies strong selection. Individuals with higher fitness, normalized within the mating range, have a higher probability of reproduction. We tracked all events during the dynamics, registering speciation, hybridization, and extinctions, and recorded the ancestry of all species.

\subsection{Diversification rates and species richness}

We simulated the evolution of populations under various strengths of selection, $\sigma_{w}$. In the non-interacting scenario (NI), the mito-nuclear distance is not considered for reproduction (equivalent to $\sigma_{w} \rightarrow \infty$ ). Selection was simulated with $\sigma_{w}$ ranging from 0.175 (weak selection) to 0.025 (strong selection). Results are shown for 50 independent realizations for each set of parameters. Starting from a population of identical individuals, all simulations are characterized by a period of rapid increase in species numbers (a radiation period), followed by relaxation to a steady-state species richness. During the dynamics, all diversification events were registered (Fig. 2a). The populations were simulated for $T=2,000$ generations, a time longer than necessary for the completion of the radiation. The equilibrium, defined by the moment when the number of species reaches a plateau, was reached after about $T=500$ generations in all cases (Fig. 2b). Selection had the clear effect of reducing the number of species in equilibrium and introducing a delay in the radiation process.

We define a speciation event, or branching, as the moment when a species splits into two or more reproductively isolated groups. The lifetime of the mother species is considered over at this point and the species disappears as two (or more) sister species are born, with a positive net balance in the number of species. Species also disappear by extinction, when the population becomes very small and eventually does not produce descendants due to random ecological drift and by the accumulation of mito-nuclear incompatibilities that lead to low fitness and low fecundity; or by hybridization when two or more species reestablish gene flow. When species 


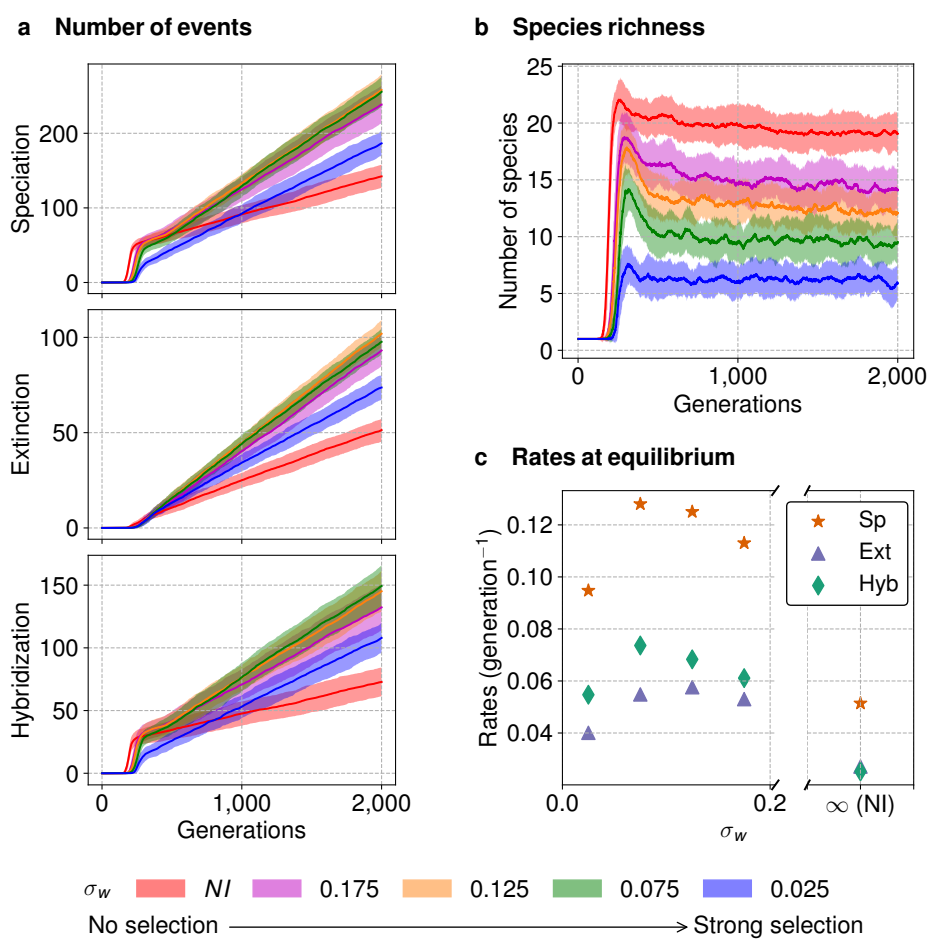

Figure 2: Diversification events and species richness. a, Accumulated number of speciation, extinction, and hybridization events over time. b, Species richness as function of time. c, Rates of speciation (Sp), extinction (Ext), and hybridization (Hyb) obtained from regression of a in equilibrium $(T>500)$. Results are shown for varying strength of the mito-nuclear selection $\sigma_{w}$ (smaller $\sigma_{w}$ implies stronger selection) and in the absence of selection (NI). Solid lines represent the ensemble mean over 50 independent realizations; and the shadowed area represents one standard deviation from the mean. Selection decreases the number of species at equilibrium, but it increases all rates of diversification events in steady-state, with maximum rates occurring at intermediary values of $\sigma_{w}$.

hybridize, we consider that the more abundant species persists while the other one disappears as it merges. Linear regression of the number of events in equilibrium provided the rates of speciation, extinction, and hybridization (Fig. 2c). In the equilibrium, when the average number of species becomes constant, the rate at which species are born (speciation) is equal to the rate at which species die (extinction plus hybridization). In this regime, speciation, extinction and hybridization rates per species increase with the strength of selection (Fig. S1). However, since the number of extant species decreases with selection, the nominal rates have a maximum for intermediate values of $\sigma_{w}$, as shown in Figure 2c. The decrease in species richness, on the other hand, results from the radiation process, because selection decreases the net diversification (speciation minus extinction and hybridization) during the transient (Fig. S2).

\subsection{Species abundance distributions and lifespans}

We have seen that selection on mito-nuclear compatibility decreases the number of species in equilibrium. Because the population size is fixed, this leads to an increase in the average 
species size and it also skews the species abundance distributions (SADs) (Fig. 3a). Since our simulations resulted in an average number of species no greater than 25 , we accumulated data over several independent runs to obtain a clear signal for the expected distribution of abundances, for each value of $\sigma_{w}$. This procedure is justified because the lattice is much larger than the mating range and species' sizes are smaller than the available space. We also validated this method by testing the scalability of the simulation (see Supplementary Information, Section S2).

We accessed species abundances in generation $T=1,000$ and found a lognormal distribution of species abundances even in the presence of strong selection over mito-nuclear compatibility (Fig. 3a). Selection increased not only the mean species abundance but also the

a Species abundances
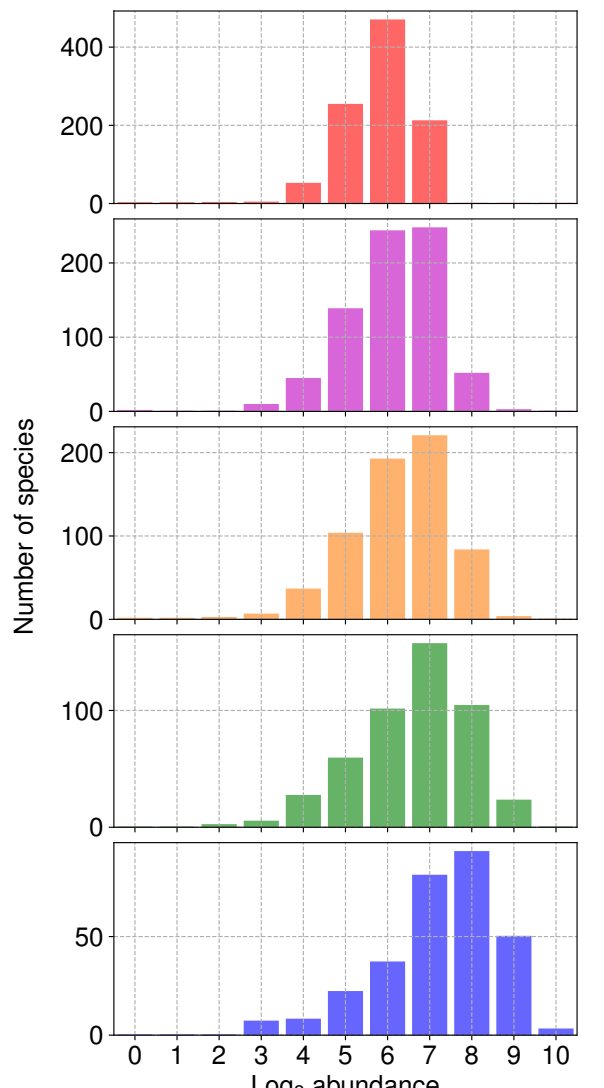

b Species lifespans
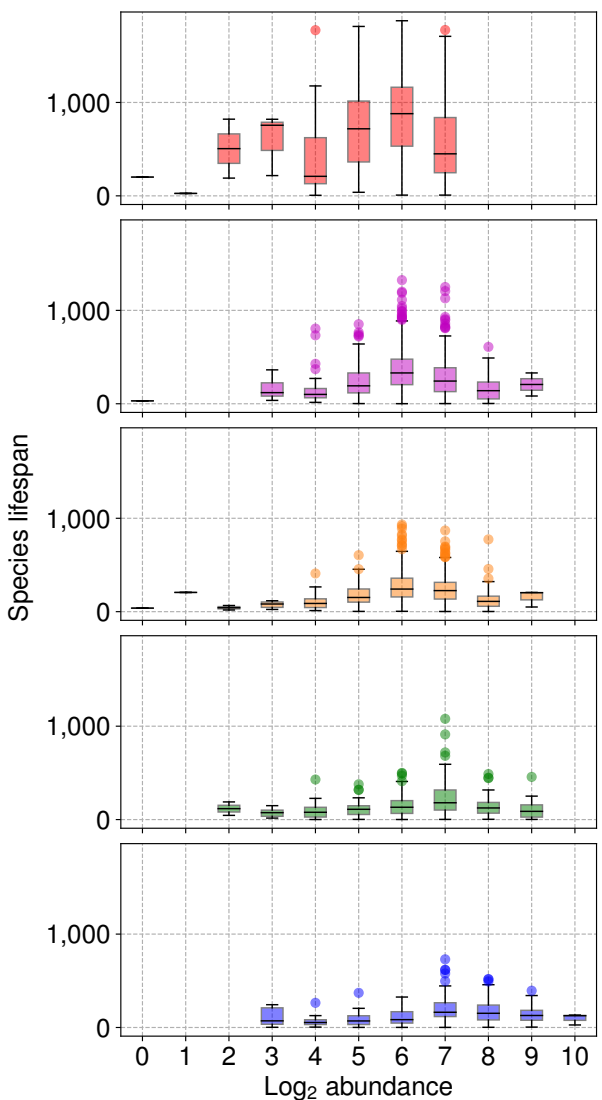

$\sigma_{w} \square N I \square 0.175 \square 0.125 \square 0.075 \square 0.025$

No selection $\longrightarrow$ Strong selection

Figure 3: Species abundances distributions (SADs) at $T=1,000$ and respective lifespans. a, SADs obtained from simulations. Species are binned into log2 abundance categories, following Preston [49]. Data were accumulated from 50 independent simulations with population size $M=1,300$. b, Respective species' lifespans within each abundance bin. Selection increases mean species' abundances and the variance of the SADs, but it tends to reduce all species lifetimes. At any point of observation, species of intermediate abundance have the longest mean lifetimes. 
variance: the histograms became wider and right-skewed (towards mono-dominance) under strong selection. The SADs are asymmetric, and, although they present a tail on the left side, low-abundant species were infrequent because speciation occurs in our model by fission of abundant species, producing relatively few rare species. SADs were stable during equilibrium, such that distributions taken at any time step after $T>500$ generations were very similar (Fig. S4).

For each species extant at time $T=1,000$, we recorded the time of its birth, the time it subsequently disappeared, and the kind of event (branching, extinction, or hybridization) that led to its eventual disappearance. Therefore, the species age and remaining lifespan were known for all species present at time $T=1,000$ (Fig. S5). The distribution of species lifespans was related with their distribution of abundances, with species binned by same abundance class (Fig. 3b). Selection tended to reduce species ages and lifetimes in all abundances classes. In all cases, species born with intermediary relative abundance had the highest longevity within their communities. In the absence of selection (NI), species lifetimes were short for low-abundant species and were equally distributed between species of intermediate or large abundance. Some of those species persisted until the end of the simulation $(T=2,000)$. Surprisingly, larger species resulting from evolution with strong selection did not tend to live longer; rather they showed drastically reduced longevity. In the non-interacting case (no selection), the most-right filled bin ( $\log 2$ abundance class 7 ) had a slight reduction of lifespan, indicating that there was also a small effect due to species size, although selection seems to be the greater cause of lifespan reduction. With regards to species ages at the time of sampling $(T=1,000)$ and their remaining lifetimes, the patterns were similar: species of intermediate abundance are the oldest and have the longest remaining lifetimes and species' ages of any abundance class decrease with selection (Fig. S5).

Species abundances at time $T=1,000$ were also related to the distribution of subsequent diversification events (Fig. 4), i.e., the mode of disappearance of each species or whether it persisted through generation $T=2,000$. Regardless of selection strength, most species had two possible endings: by branching, if it was dominant within the community, or by extinction, if it had low abundance. Remarkably, in the absence of mito-nuclear selection, a significant portion of species persisted through to generation $T=2,000$. Selection suppressed the persistence of species, even when weak $\left(\sigma_{w}=0.175\right)$. Selection also increased the rate of hybridization (note that hybridization events were counted only for low-abundant species due to our convention of considering the least abundant species involved as dying during the fusion, whereas the more 

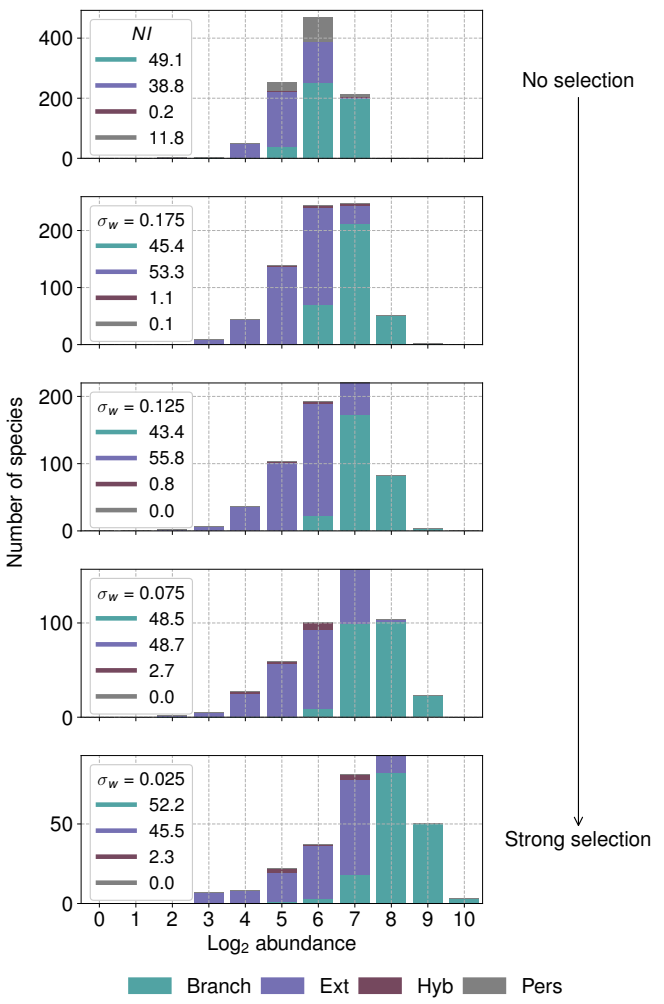

Figure 4: Long-term evolutionary fates of species that were extant at time $T=1,000$. The histograms depicts the fate of each species shown in Fig. 3a at time: by branching (Branch), extinction (Ext), or hybridization (Hyb), or persistence through time $T=2000$ (Pers), binned by log2 abundance class. The legends indicate the percentages of total species that correspond to each event category. Most species die by branching if they are dominant within the community, or by extinction if they are rare. Selection for mito-nuclear compatibility increases rates of hybridization and suppresses long-term persistence.

abundant species persists with increased abundance). Notably, species belonging to the same class of abundances had different fates, depending on whether they were under strong, weak, or no selection. For instance, a species belonging to bin 6 in the non-interacting scenario could persist for 1,000 generations ahead, while a species of the same size was most likely to go extinct in any scenario with selection. Whereas abundance class 7 was the largest one in simulations without selection, more likely to speciate, but it was not dominant and more likely to go extinct under strong selection.

\subsection{Genetic diversity of the populations}

In our model, micro-processes drive the emergence and death of species. Therefore, the analysis of genetic patterns can help to elucidate the mechanism by which mito-nuclear selection promotes or hinders diversification. We evaluated genetic diversity by measuring the nuclear genetic distance between pairs of individuals at time $T=2,000$. Mito-nuclear selection 
drastically reduced the genetic distances between individuals belonging to different species (Fig. 5a). In the absence of selection (NI), species were genetically well isolated from each other, with a prominent peak around 650 loci. Increasing the strength of selection (reducing $\left.\sigma_{w}\right)$ made species progressively more similar to each other genetically. However, the effect was opposite for conspecific individuals (Fig. 5b): under selective pressure, we observed a slightly larger nuclear divergence within a species than in the non-selective case. Notably for strong selection, several pairs of individuals had distances exceeding the genetic threshold $(G=75)$ required for mating. In this case, species cohesion was kept by ongoing gene flow.

The patterns of diversity between different species were similar for the mtDNA content (Fig. S6a) and is explained by the effect of the mito-nuclear selection on reducing both nuclear and mitochondrial substitutions. When reproduction can only occur in individuals with high

a Interspecies diversity

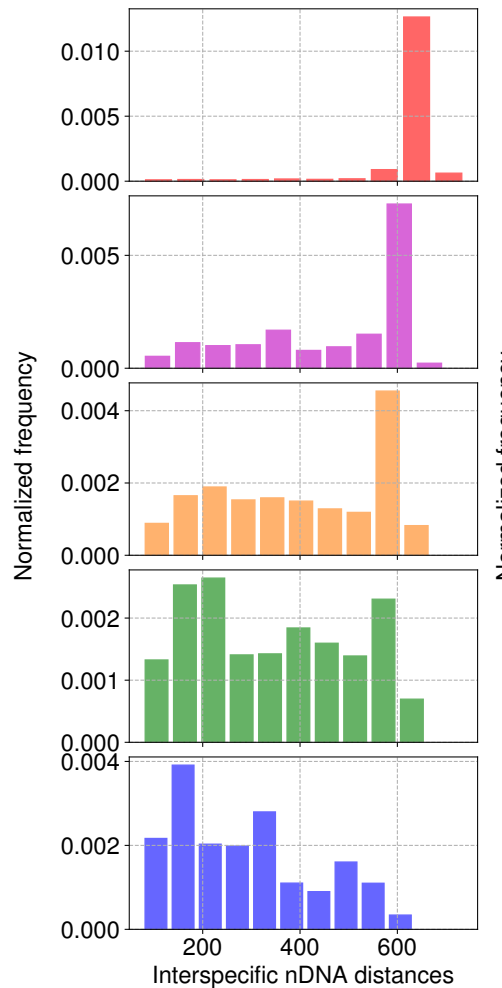

b Intraspecies diversity
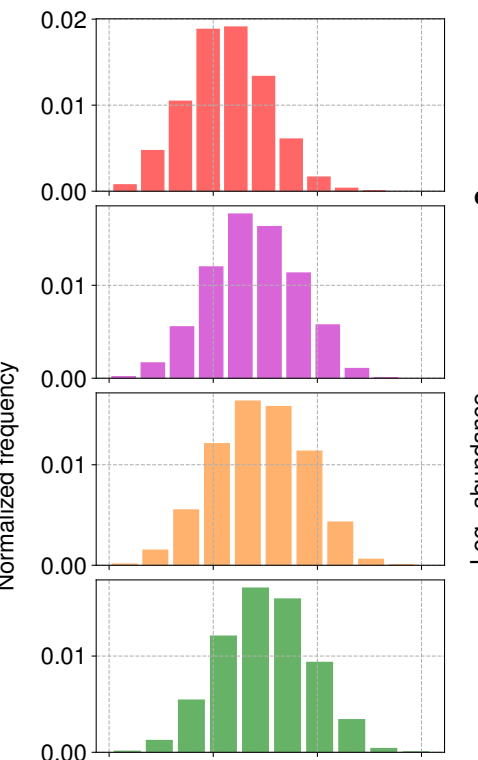

0.00

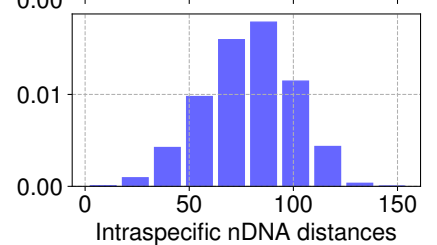

Intraspecies diversity vs abundance

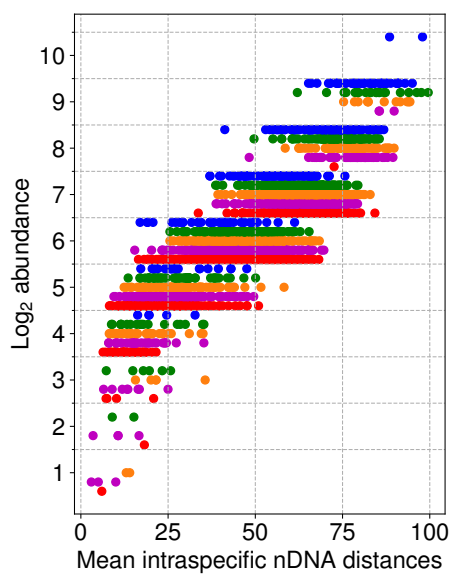

$$
\begin{aligned}
& \sigma_{w} \square \mathrm{NI} \square 0.175 \square 0.125 \square 0.075 \square \\
& \text { No selection } \square \text { Strong selection }
\end{aligned}
$$

Figure 5: Genetic diversity within and between species. The histograms depict the nDNA distances between pairs of individuals that belong to different species (a), or are conspecific (b) after $T=2,000$ generations. We also show the mean nDNA distance within each species, plotted against the species abundance classes (c). nDNA distances between different species are much lower under strong selection, while the nDNA distances within species are slightly higher. The latter effect is largely due to species abundances: as shown in c, more abundant species allow for greater intraspecific diversity, independent of selection strength. 
fitness (low mito-nuclear distance), fixation of a mutation in any of the genome is hampered, because it must be followed by a mutation in the corresponding site of the interacting pair to maintain the mito-nuclear compatibility. As a result, interacting sites had lower substitution rates in both the mitochondrial and nuclear genomes (Fig. S7). Within a species, the mean nDNA distance within species is positively correlated to the species' abundance (Fig. 5c), independent of the selection strength. As selection increased species abundances, the effect on the population was to increase the nDNA distances within each species. Therefore, the mito-nuclear interaction promoted the intraspecies genetic diversity but reduced the global diversity in the community.

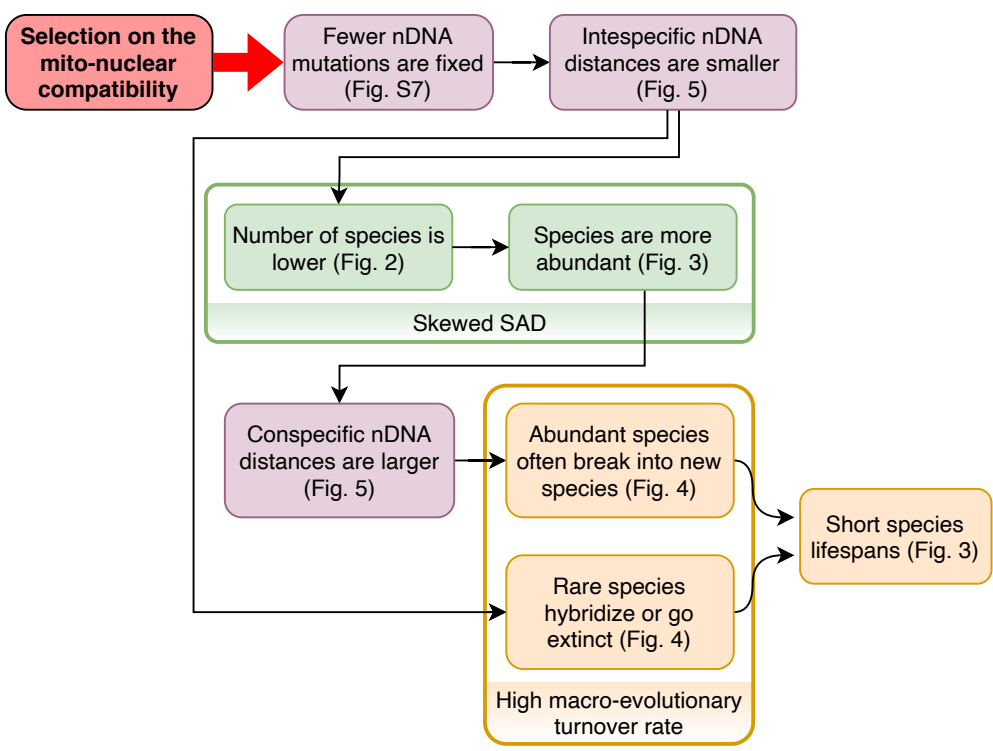

\begin{tabular}{|l|c|c|}
\cline { 2 - 3 } \multicolumn{1}{c|}{} & $\begin{array}{c}\text { Absence of } \\
\text { selection } \\
\left(\sigma_{w} \rightarrow \infty\right)\end{array}$ & $\begin{array}{c}\text { Strong } \\
\text { selection } \\
\left(\sigma_{w} \ll 1\right)\end{array}$ \\
\hline Species richness & High & Low \\
Extant species & Old & Young \\
Species lifespan & Long-lived & Short-lived \\
Speciation rate & Low & High \\
Extinction rate & Low & High \\
Hybridization rate & Low & High \\
\hline
\end{tabular}

Figure 6: From selection at the individual level to ecological and macro-evolutionary patterns. The flow chart (left) summarizes the cascade of events connecting selection on mito-nuclear compatibility within individuals to its effects on populations, species, and evolutionary turnover. Colors represent the scale of effects: genetic (purple), ecological (green), and evolutionary (orange). The table (right) compares the equilibrium eco-evolutionary patterns in populations under weak versus strong selection. These predictions are qualitatively similar to empirical ecological and evolutionary patterns in tropical (weak selection) versus temperate zones (strong selection).

A chain of effects connects selection on mito-nuclear genetic compatibility within an individual level to skewed species abundance distributions in a community, shorter species lifespans, and elevated macro-evolutionary turnover (chart at Fig. 6). Strong selection promotes the conservation of the nuclear genetic content in the population because mutations accumulated more slowly. Consequently, the disruption of gene flow is hindered, and the number of species diminished. With a reduced number of species, they are naturally more abundant and support more diversity, favoring evolutionary branching events. Therefore, the speciation rate increases, and abundant species have reduced lifetimes. Low-abundant 
(non-dominant) species are also short-lived: due to the shorter interspecies distances, neighbor species could easily incorporate individuals from these small species, causing abundance fluctuations that increase the probability of extinction. The reduced genetic distances between different species also promotes hybridization, with sister species merging more easily after speciation. As a global result, communities under mito-nuclear selection were characterized by a low number of species, skewed SADs, short species lifespans and high rates of speciation, extinction, and hybridization, i.e., high turnover rate.

\section{Discussion}

We have investigated how micro-evolutionary processes impact ecological and macro-evolutionary patterns in a model with selection for mito-nuclear compatibility. Speciation, hybridization, and extinction are emergent phenomena in our model, byproducts of changes in allele frequency in the populations assisted by spatial and genetic mating restrictions. We have shown that mito-nuclear selection at the individual level triggers a succession of effects in populations and species that result in low species richness in the equilibrium, low species lifespans, and high evolutionary turnover (Fig. 6). We investigated the mechanisms by which selection induces these effects, and how they are correlated across biological scales.

Our results show significant correspondence to empirical latitudinal gradients of diversity. The conventional views claim that biodiversity hot spots should sustain more speciation events than regions with few species $[19,50,51]$, creating a latitudinal gradient of species richness. Speciation would be more frequent in the tropics due to faster evolution of reproductive isolation, driven by higher metabolic rates (due to environmental factors, such as temperature) and greater ecological opportunities, among other possible causes $[23,52]$. A large number of species would also increase the likelihood of extinction due to competition [52-54]. However, the observation of higher speciation rates in temperate zones contradicts this conventional view and establishes unexpected links between species richness, evolutionary trajectories and turnover [20-25]. As one moves from the tropics to temperate areas, speciation rates increase, followed by decreasing ages of sister species $[18,20,23]$. Despite having a high speciation rate, the low number of species in these regions can be explained by increased extinction and hybridization rates. We observe a qualitative similarity between populations evolved under strong selection in our model 
and empirical patterns in temperate zones; and they differ from simulations under weak or no selection which are similar to empirical populations in the tropics (see table in Fig. 6). Temperate zones, generally regarded as harsh environments $[21,22,26]$, are reasonably modelled by assuming strong selection, due to the need for mito-nuclear compatibility for metabolic function, which is most critical in harsh temperate habitats with low resources. Experimental validation of such a conjecture is, however, challenging because extinction rates are difficult to measure by molecular data, and fossils records are incomplete $[22,55]$.

The genetic patterns in our simulated populations reflect the primary causes of variation in diversification rates. Mito-nuclear selection strongly reduced the nuclear genetic distance between species; however, intraspecies nDNA diversity was slightly elevated (Fig. 5). The maintenance of mito-nuclear compatibility slows the fixation of mutations for both nDNA and mtDNA (Fig. S7). Substitutions occurred more slowly, delaying and prolonging the radiation stage, and in smaller numbers, promoting the conservation of the genetic content in the population. Consequently, the disruption of gene flow was hindered, resulting in a low number of species. When species were fewer in number, they were more abundant, helping to establish greater intraspecific genetic diversity (Fig. 5c). These species branched more frequently, shortening their lifetimes and resulting in a high speciation rate despite the lower substitution rate of the nuclear alleles. Notably, the average rate of substitutions was not positively correlated with the speciation rate, which has previously been proposed to be a driver of biodiversity (revised by [19]).

In contrast to rare species that are more extinction-prone, abundant species are expected to be old and long-lived, i.e. more robust to the ecological drift $[2,10]$. However, we found that selection reduced species' lifespans even though they were abundant (Fig. 3). We conclude that, for abundant species, the intrinsic robustness to the ecological drift was opposed by purifying selection against mito-nuclear incompatibilities that rapidly decreased individual fitness as mutations accumulate. Frequent speciation by species' fission was the mechanism responsible for the death of abundant species under strong selection, which explains the relationship between the short lifetime of abundant species and the corresponding high speciation rate [12]. Genetic divergence between species is generally greater in the tropics than in high latitudes, usually explained by the long age of tropical species [23], and intraspecific divergences are higher at temperate zones, associated with recent speciation rates $[21,22,31,32,56]$. Our results suggest that the process of the build-up of genetic diversity 
may be the cause, rather than the consequence, of species being older or younger. Furthermore, the model behavior under strong selection contradicts the prediction that new species should be rare when a species is dominant ( [10], according to the neutral theory).

A strong positive correlation between environmental harshness and the differentiation within species $[21,22]$ suggests that speciation is frequent in those regions, which is supported by the observation of young ages [32], since most species are susceptible to extinction. Cutter and Gray [26] scrutinized how harsh environments promote the recurrent fragmentation of species in space, which may sequentially develop reproductive barriers $[9,33]$. Those incipient species would evolve through local adaptation and ecological speciation, and, from the nature of these processes, they are more prone to hybridization. Our model produced similar patterns in a process driven by stabilizing (purifying) selection, in contrast to the suggested mechanism of ecological speciation (based on divergent selection), which shows that the same macro-evolutionary pattern can result from distinct underlying processes.

\section{Methods}

\subsection{Model}

We use an individual-based model (IBM) of speciation adapted from [5], following the same methodology of our previous work [38]. We consider a spatially distributed population evolving in the absence of geographic barriers under the influence of mutation, genetic drift, and dispersion. Reproductive isolation results from two types of restrictions to mating: (i) genetic compatibility, a weak form of assortative mating, requiring a threshold genetic distance $G$ between mating pairs, and (ii) spatial proximity of the mating pair, which must be at maximum distance $S$ from each other, simulating parapatry. We call the area of radius $S$ around an individual its mating neighborhood. Species emerge from the dynamics as groups of individuals connected by gene flow and separated from all others by the genetic mating restriction $G$. Not all individuals of a species need to be compatible since gene flow can be established through intermediary individuals. In other words, species correspond to the components of a network where individuals (nodes) are connected if their nuclear genetic distance is less than or equal to $G$. The model predicts patterns of biodiversity, such as species abundance distributions (SADs) and species-area relationships [5], similar to previous neutral models [1-3].

The dynamics describes the evolution of a population with $M$ individuals, represented by 
single copies of their nuclear and mitochondrial genomes, modeled as binary strings with sizes $B$ and $B_{M}$ respectively. Sexes are separated and individuals are randomly distributed over a square area of side $L$ (see Fig. 1a) with periodic boundary conditions (individuals can, by chance, occupy the same lattice site). Generations do not overlap and the population is fully replaced by the offspring. Each individual has a probability $P$ to reproduce and $Q=1-P$ of dying without leaving offspring. For reproduction, a mating partner is randomly chosen among genetically compatible individuals in its mating neighborhood. The nuclear genetic distance $D_{n}$ between individuals is defined as the Hamming distance between their nuclear genomes, and compatibility requires $D_{n} \leq G$. If a genetically compatible mate is found within the mating neighborhood of the 'focal' individual, an offspring is produced with locus by locus recombination of the nuclear genomes, followed by mutation with probability $\mu$ per locus. The mtDNA is inherited from the mother, with mutations with probability $\mu_{M}$ (Fig. 1a). The offspring is placed at the site of the focal individual or, with diffusion probability $D$, in one of $S_{D}$ randomly chosen nearest neighbor sites. Note that individuals can generate more than one offspring: when selected as mating partner by their neighbors or when playing the role of focal individual. If the focal individual cannot find a compatible mate in its mating neighborhood, it expands his search area to radius $S+1$ and then to $S+2$. If no compatible mate is found in the extended area, or if the individual dies without reproducing (probability $Q$ ), another individual is randomly selected within its original mating neighborhood to reproduce in its place, keeping the population size constant. On rare occasions, however, due to population fluctuations, the mating neighborhood might be empty and no replacement can be found. In these cases, mating does not occur and the population size decreases by one. In order to restore the population to its carrying capacity $M$, we allow individuals to have two offspring in the next generation if their mating neighborhoods have densities below $60 \%$ of the expected average value $M / L^{2}$. This procedure introduces small fluctuations in population size.

We compare the neutral process, when mito-nuclear selection is absent, with the scenario where mito-nuclear compatibility promotes fitness differences in the population, testing different levels of selective pressure. The mito-nuclear distance $d$ measures the compatibility between the mtDNA and the corresponding loci of nDNA, assigned to each individual. The individual's fitness $w$ depends on $d$ according to a Gaussian function with width $\sigma_{w}$, which quantifies the selective strength of the mito-nuclear interaction (Fig. 1b). Selection over mito-nuclear compatibility changes the probability of an individual to reproduce, i.e., it affects 
its fecundity. The focal individual has its chance for reproduction modified by its fitness as follows: first the fitness of all individuals in the population are computed as $w(i)=e^{-d(i)^{2} / 2 \sigma_{w}^{2}}$; second, $Q$ for individual $i$ is modified to $Q_{w}(i)=2 Q\left(w_{\max }-w(i)\right) /\left(w_{\max }-w_{\min }\right)$ where $w_{\max }$ and $w_{\min }$ are the maximum and minimum fitness in the population, respectively. Individuals with $w(i)=w_{\max }$ have $Q_{w}(i)=0$ and probability of reproducing $P_{w}=1-Q_{w}=1$. Those with $w(i)=w_{\min }$ have $Q_{w}(i)=2 Q$ and $P_{w}=1-2 Q$. Therefore, individuals with low fitness still have a small chance of reproducing. If the individual dies without reproducing, another one is selected in its mating neighborhood, also according to fitness, to reproduce in its place, keeping the total population constant. Finally, when searching for a mate, the probability of selecting a partner is again proportional to the fitness of the compatible individuals in the mating neighborhood, simulating local competition.

For the analysis, we considered the following set of parameters (unless said otherwise): a population of $M=1,300$ individuals randomly distributed in $(100 \times 100)$ lattice with reflecting boundary conditions; the nuclear genome length is $B=1,500$, with mutation rate $\mu=0.00025$, genetic similarity threshold $G=75=0.05 B$ and mating range $S=5$. Population density is 0.13 , indicating that there are many empty sites in the lattice; the average number of individuals in the mating neighborhood is 10 . The mitochondrial genome length is $B_{M}=500$, with mutation rate $\mu_{M}=3 \mu$. We start from an initial condition where all individuals are identical, therefore emulating a radiation process. We also fixed $D=0.02$ and $S_{D}=20$. In this work we simulate the evolutionary process for $T=2,000$ generations. We fixed $Q=0.37 \approx e^{-1}$ as the neutral probability of dying without leaving offspring (equal to the chance of not selecting one particular individual in $M$ trials with replacement [57]). We have run 50 independent realizations of each scenario and present the population averaged or accumulated measurements.

\subsection{Processes of diversification}

Throughout the evolutionary dynamics, recombination and mutation change allele frequencies in the population and, combined with the genetic and spatial restrictions for mating, reproductively isolated species can emerge. New species are born from an abundant one that eventually divides into smaller clusters with disrupted gene flow. Due to the model's stochasticity, species abundances and species richness fluctuate randomly (ecological drift). The number of individuals of a species can progressively diminish until no offspring is left to the next generation, due to lack of compatible partners in the mating neighborhood or by 
chance, and the species is extinct. Also, genetically close species can merge by the reestablishment of the gene flow due to mutations, hybridizing. Therefore we identify three classes of events in which a species can disappear when registering the evolutionary history of the populations: speciation or branching: when an abundant species breaks into two or more new species. The mother species is considered dead and new species are born, adding to the number of living species; extinction: when the number of individuals of the species progressively diminishes until it reaches a null abundance; and hybridization: when two or more species merge, reestablishing the gene flow. In this case we adopted the protocol of maintaining the largest species and consider the smaller species dead, absorbed by the largest one.

Branching events increase species richness, while extinction and hybridization decrease the number of extant species, reducing biodiversity. Hybridization is an emergent event in our model and only cause loss of diversity. In nature, however, hybridization may increase species richness inducing the evolution of reproductive barriers [58]. Here we emulated the loss of diversity due to hybridization from lineage fusion, speciation reversal [59], and hybrid breakdown [60,61]. We included in this category events of failed speciation attempts (when two or more recent sister species merge back) and events of reticulate evolution (when there are two speciation events in a row and a species fuses with the one with the most distant ancestor)

\subsection{Species abundances distributions}

In our simulations the number of species in equilibrium $(T \geq 500)$ ranged from about 20 in the non-interacting case to about 5 for strong selection (Fig. 2b). To generate abundance distribution plots with statistical significance, we have run 50 independent simulations for each set of parameters and accumulated the results. This increased the number of species in the histograms to about 1,000 for the non-interacting case and 100 for the case of strong selection. Section S2 of Supplemental Materials justifies the validity of this procedure.

To generate the species abundances histograms, the data was binned as suggested by Preston [62] in abundances classes $(\log 2)$. There are several methods for choosing the bins boundaries [49], and we followed the method adopted by [1,63]: bins were built by using the powers of two as the centre of bins. The boundaries then come as $2^{n-1 / 2}$ and $2^{n+1 / 2}$. Thus, bin $=0$ stores species with 1 individual, bin $=1$ counts species with 2 individuals, on $b i n=2$ species with 3,4 and 5 individuals, and so on. 


\section{Code availability}

All simulations are run in Fortran, and the scripts wrapped in Python are available in the GitHub repository at https://github.com/deborapr/mito-nuclear-speciation.

\section{References}

1. Hubbell, S. P. The Unified Neutral Theory of Biodiversity and Biogeography (MPB-32) (Princeton University Press, Princeton, 2001).

2. Volkov, I., Banavar, J. R., Hubbell, S. P. \& Maritan, A. Neutral theory and relative species abundance in ecology. Nature 424, 1035-1037 (2003).

3. Volkov, I., Banavar, J. R., Hubbell, S. P. \& Maritan, A. Patterns of relative species abundance in rainforests and coral reefs. Nature 450, 45-49 (2007).

4. McGill, B. J. et al. Species abundance distributions: moving beyond single prediction theories to integration within an ecological framework. Ecology Letters 10, 995-1015 (2007).

5. de Aguiar, M. A. M., Baranger, M., Baptestini, E. M., Kaufman, L. \& Bar-Yam, Y. Global patterns of speciation and diversity. Nature 460, 384 (2009).

6. O'Dwyer, J. P. \& Green, J. L. Field theory for biogeography: A spatially explicit model for predicting patterns of biodiversity. Ecology Letters 13, 87-95 (2010).

7. Chisholm, R. A. \& Pacala, S. W. Niche and neutral models predict asymptotically equivalent species abundance distributions in high-diversity ecological communities. Proceedings of the National Academy of Sciences of the United States of America 107, 15821-15825 (2010).

8. Mutshinda, C. M., O'Hara, R. B. \& Woiwod, I. P. What drives community dynamics? Proceedings of the Royal Society B: Biological Sciences 276, 2923-2929 (2009).

9. Rosindell, J., Cornell, S. J., Hubbell, S. P. \& Etienne, R. S. Protracted speciation revitalizes the neutral theory of biodiversity. Ecology Letters 13, 716-727 (2010).

10. Chisholm, R. A. \& O’Dwyer, J. P. Species ages in neutral biodiversity models. Theoretical Population Biology 93, 85-94 (2014).

11. Nee, S. The neutral theory of biodiversity: do the numbers add up? Functional Ecology 19, 173-176 (2005). 
12. Ricklefs, R. E. A comment on Hubbell's zero-sum ecological drift model. Oikos 100, 185-192 (2003).

13. Etienne, R. S., Apol, M. E. F., Olff, H. \& Weissing, F. J. Modes of speciation and the neutral theory of biodiversity. Oikos 116, 241-258 (2007).

14. Davies, T. J., Allen, A. P., Borda-de Água, L., Regetz, J. \& Melián, C. J. Neutral biodiversity theory can explain the imbalance of phylogenetic trees but not the tempo of their diversification. Evolution 65, 1841-1850 (2011).

15. Rosindell, J., Harmon, L. J. \& Etienne, R. S. Unifying ecology and macroevolution with individual-based theory. Ecology Letters 18, 472-482 (2015).

16. Thuiller, W. et al. A road map for integrating eco-evolutionary processes into biodiversity models. Ecology Letters 16, 94-105 (2013).

17. Hillebrand, H. On the generality of the latitudinal diversity gradient. American Naturalist 163, 192-211 (2004).

18. Kennedy, J. D. et al. Into and out of the tropics: The generation of the latitudinal gradient among New World passerine birds. Journal of Biogeography 41, 1746-1757 (2014).

19. Mittelbach, G. G. et al. Evolution and the latitudinal diversity gradient: Speciation, extinction and biogeography. Ecology Letters 10, 315-331 (2007).

20. Weir, J. T. \& Schluter, D. The latitudinal gradient in recent speciation and extinction rates of birds and mammals. Science 315, 1574-1576 (2007).

21. Botero, C. A., Dor, R., McCain, C. M. \& Safran, R. J. Environmental harshness is positively correlated with intraspecific divergence in mammals and birds. Molecular Ecology 23, 259-268 (2014).

22. Weir, J. T. Environmental harshness, latitude and incipient speciation. Molecular Ecology 23, 251-253 (2014).

23. Schluter, D. \& Pennell, M. W. Speciation gradients and the distribution of biodiversity. Nature 546, 48-55 (2017).

24. Harvey, M. G. et al. The evolution of a tropical biodiversity hotspot. Science 370, 1343-1348 (2020). 
25. Rabosky, D. L. et al. An inverse latitudinal gradient in speciation rate for marine fishes. Nature 559, 392-395 (2018).

26. Cutter, A. D. \& Gray, J. C. Ephemeral ecological speciation and the latitudinal biodiversity gradient. Evolution 70, 2171-2185 (2016).

27. Benton, M. J. The Red Queen and the Court Jester: Species Diversity and the Role of Biotic and Abiotic Factors Through Time. Science 323, 728-732 (2009).

28. Ezard, T. H., Aze, T., Pearson, P. N. \& Purvis, A. Interplay between changing climate and species' ecology drives macroevolutionary dynamics. Science 332, 349-351 (2011).

29. Morlon, H. Phylogenetic approaches for studying diversification. Ecology Letters 17, 508-525 (2014).

30. Rabosky, D. L. \& Matute, D. R. Macroevolutionary speciation rates are decoupled from the evolution of intrinsic reproductive isolation in Drosophila and birds. Proceedings of the National Academy of Sciences 110, 15354-15359 (2013).

31. Harvey, M. G. et al. Positive association between population genetic differentiation and speciation rates in New World birds. Proceedings of the National Academy of Sciences of the United States of America 114, 6328-6333 (2017).

32. Smith, B. T., Seeholzer, G. F., Harvey, M. G., Cuervo, A. M. \& Brumfield, R. T. A latitudinal phylogeographic diversity gradient in birds. PLOS Biology 15, e2001073 (2017).

33. Li, J., Huang, J.-P., Sukumaran, J. \& Knowles, L. L. Microevolutionary processes impact macroevolutionary patterns. BMC Evolutionary Biology 18, 123 (2018).

34. Costa, C. L. N. et al. Signatures of Microevolutionary Processes in Phylogenetic Patterns. Systematic Biology 68, 131-144 (2018).

35. Louca, S. \& Pennell, M. W. Extant timetrees are consistent with a myriad of diversification histories. Nature 580, 502-505 (2020).

36. Rosindell, J. \& Harmon, L. J. A unified model of species immigration, extinction and abundance on islands. Journal of Biogeography 40, 1107-1118 (2013). 
37. Etienne, R. S. \& Rosindell, J. Prolonging the past counteracts the pull of the present: Protracted speciation can explain observed slowdowns in diversification. Systematic Biology 61, 204-213 (2012).

38. Princepe, D. \& De Aguiar, M. A. M. Modeling Mito-nuclear Compatibility and Its Role in Species Identification. Systematic Biology 70, 133-144 (2021).

39. Bar-Yaacov, D., Blumberg, A. \& Mishmar, D. Mitochondrial-nuclear co-evolution and its effects on OXPHOS activity and regulation. Biochimica et Biophysica Acta - Gene Regulatory Mechanisms 1819, 1107-1111 (2012).

40. Barreto, F. S. et al. Genomic signatures of mitonuclear coevolution across populations of Tigriopus californicus. Nature Ecology and Evolution 2, 1250-1257 (2018).

41. Hill, G. E. Mitonuclear coevolution as the genesis of speciation and the mitochondrial DNA barcode gap. Ecology and Evolution 6, 5831-5842 (2016).

42. Lima, T. G., Burton, R. S. \& Willett, C. S. Genomic scans reveal multiple mito-nuclear incompatibilities in population crosses of the copepod Tigriopus californicus. Evolution (2019).

43. Sunnucks, P., Morales, H. E., Lamb, A. M., Pavlova, A. \& Greening, C. Integrative Approaches for Studying Mitochondrial and Nuclear Genome Co-evolution in Oxidative Phosphorylation. Frontiers in genetics 8, 25 (2017).

44. Wolff, J. N., Ladoukakis, E. D., Enríquez, J. A. \& Dowling, D. K. Mitonuclear interactions: evolutionary consequences over multiple biological scales. Philosophical Transactions of the Royal Society B: Biological Sciences 369, 20130443 (2014).

45. Hill, G. E. Mitonuclear Ecology (Oxford University Press, Oxford, U.K., 2019).

46. Koch, R. E. et al. Integrating Mitochondrial Aerobic Metabolism into Ecology and Evolution. Trends in Ecology \& Evolution 36, 321-332 (2021).

47. Ballard, J. W. O. \& Rand, D. M. The Population Biology of Mitochondrial DNA and Its Phylogenetic Implications. Annual Review of Ecology, Evolution, and Systematics 36, $621-642(2005)$. 
48. Avise, J. C. et al. Intraspecific Phylogeography: The Mitochondrial DNA Bridge Between Population Genetics and Systematics. Annual Review of Ecology and Systematics 18, 489-522 (1987).

49. Gray, J. S., Bjørgesæter, A. \& Ugland, K. I. On plotting species abundance distributions. Journal of Animal Ecology 75, 752-756 (2006).

50. Emerson, B. C. \& Kolm, N. Species diversity can drive speciation. Nature 434, 1015-1017 (2005).

51. Emerson, B. C. \& Kolm, N. Is speciation driven by species diversity? (Reply). Nature 438, E2-E2 (2005).

52. Allen, A. P. \& Gillooly, J. F. Assessing latitudinal gradients in speciation rates and biodiversity at the global scale. Ecology letters 9, 947-954 (2006).

53. MacArthur, R. H. \& Wilson, E. O. The Theory of Island Biogeography (Princeton University Press, Princeton, 1967).

54. Yamaguchi, R., Iwasa, Y. \& Tachiki, Y. Recurrent speciation rates on islands decline with species number. Proceedings of the Royal Society B: Biological Sciences 288, 20210255 (2021).

55. Rabosky, D. L. Extinction rates should not be estimated from molecular phylogenies. Evolution 64, 1816-1824 (2010).

56. Araújo, M. S. \& Costa-Pereira, R. Latitudinal gradients in intraspecific ecological diversity. Biology Letters 9 (2013).

57. de Aguiar, M. A. M. Speciation in the Derrida-Higgs model with finite genomes and spatial populations. Journal of Physics A: Mathematical and Theoretical 50, 85602 (2017).

58. Abbott, R. et al. Hybridization and speciation. Journal of Evolutionary Biology 26, 229-246 (2013).

59. Kearns, A. M. et al. Genomic evidence of speciation reversal in ravens. Nature Communications 9, 906 (2018). 
60. Han, K.-L. \& Barreto, F. S. Pervasive Mitonuclear Coadaptation Underlies Fast Development in Interpopulation Hybrids of a Marine Crustacean. Genome Biology and Evolution 13 (2021).

61. Burton, R. S., Pereira, R. J. \& Barreto, F. S. Cytonuclear Genomic Interactions and Hybrid Breakdown. Annual Review of Ecology, Evolution, and Systematics 44, 281-302 (2013).

62. Preston, F. W. The Commonness, And Rarity, of Species. Ecology 29, 254-283 (1948).

63. Williamson, M. \& Gaston, K. J. The lognormal distribution is not an appropriate null hypothesis for the species-abundance distribution. Journal of Animal Ecology 74, 409-422 (2005). 


\section{Supplementary Files}

This is a list of supplementary files associated with this preprint. Click to download.

- SupplementaryInformation.pdf 